\title{
[3+2] Cycloaddition of Tosylmethyl Isocyanide with Styrylisoxazoles: Facile Access to Polysubstituted 3-(Isoxazol-5-yl)pyrroles
}

\author{
Xueming Zhang ${ }^{1}$, Xianxiu $\mathrm{Xu}^{2}$ and Dawei Zhang ${ }^{1, *}$ \\ 1 College of Chemistry, Jilin University, Changchun 130012, China; xue15603228979@foxmail.com \\ 2 College of Chemistry, Chemical Engineering and Materials Science, Key Laboratory of Molecular and \\ Nano Probes, Ministry of Education, Shandong Normal University, Jinan 250014, China; \\ xuxx677@sdnu.edu.cn \\ * Correspondence: z_dw@jlu.edu.cn; Tel.: +86-431-878-36471
}

Received: 16 June 2017; Accepted: 3 July 2017; Published: 7 July 2017

\begin{abstract}
A facile access to polysubstituted 3-(isoxazol-5-yl)pyrroles was developed through [3+2] cycloaddition of tosylmethyl isocyanide (TosMIC) and styrylisoxazoles. In the presence of $\mathrm{KOH}$, various styrylisoxazoles reacted smoothly with tosylmethyl isocyanide and analogs to deliver a wide range of 3-(isoxazol-5-yl)pyrroles at ambient temperature. This transformation is operationally simple, high-yielding, and displays broad substrate scope.
\end{abstract}

Keywords: isoxazol-5-ylpyrroles; [3+2]cycloaddition; TosMIC; 3-methyl-4-nitro-5-styrylisoxazoles

\section{Introduction}

Pyrrole derivatives are one of the most relevant heterocycles with important biological activities, which includes antitumour, antibacterial, antiviral, anti-inflammatory, antioxidative, and are also widely used in organic synthesis as key heterocycles and/or intermediates for the preparation of natural compounds and related structures, and molecular sensors [1]. In this context, isoxazole substituted pyrroles are present as the core substructure in some meaningful compounds, such as isoxazolylpyrroles I and II are inhibitors to oral and mouth cancer cell and the activators to cellular tumor antigen p53 [2,3]. Isoxazolylpyrroles III and IV are the key intermediates in the synthesis of bioactive prodiginines natural products and their congeners, and the precursors structures of phosphodiesterase inhibitors PDE-I and PDE-II, which inhibitory activity toward cyclic adenosine- $3^{\prime}, 5^{\prime}$-monophosphate phosphodiesterase, respectively [4,5]. Isoxazolylpyrroles $\mathbf{V}$ is a receptor for recognition and sensing purposes in aprotic solvents [6,7]. (Figure 1).

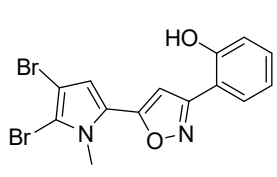

I

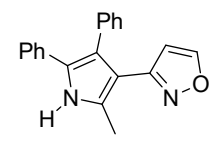

II

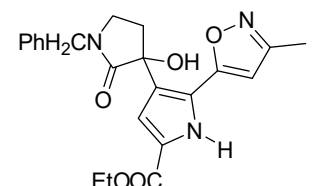

III

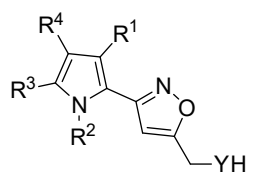

IV

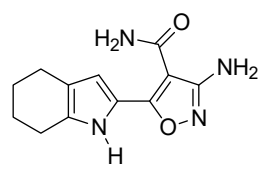

V

Figure 1. Examples of biologically active, isoxazole-substituted pyrrole derivatives. 
In the view of the applications of isoxazole substituted pyrrole, some synthetic methods have been developed for their preparation. Among these known synthetic approaches, two main strategies are shown as follows: one is the construction of isoxazole ring from starting materials containing pyrrole ring, such as the 1,3-dipolar cycloaddition reaction of 1,5-diphenyl-1,4-pentadien-3-one with nitrile oxides in the presence of chloramine-T reported by Padmavathi et al. (Scheme 1, Equation (1)) [8] , or [3+2]-cycloadditions of enaminone and hydroxylamine hydrochloride reported by Gomha et al. (Scheme 1, Equation (2)) [3]. In contrast, another synthetic strategy is through the construction of pyrrole ring from starting materials containing isoxazole ring, including the four-component coupling reaction of a functionalized silane, a nitrile, an aldehyde, and trimethylsilylcyanide by $\mathrm{Yb}(\mathrm{OTf})_{3}$-catalyzed reported by Konakahara et al. (Scheme 1, Equation (3)) [9]. Despite these achievements, the development of novel methods for the convenient synthesis of the isoxazole substituted pyrroles is still of great interest.

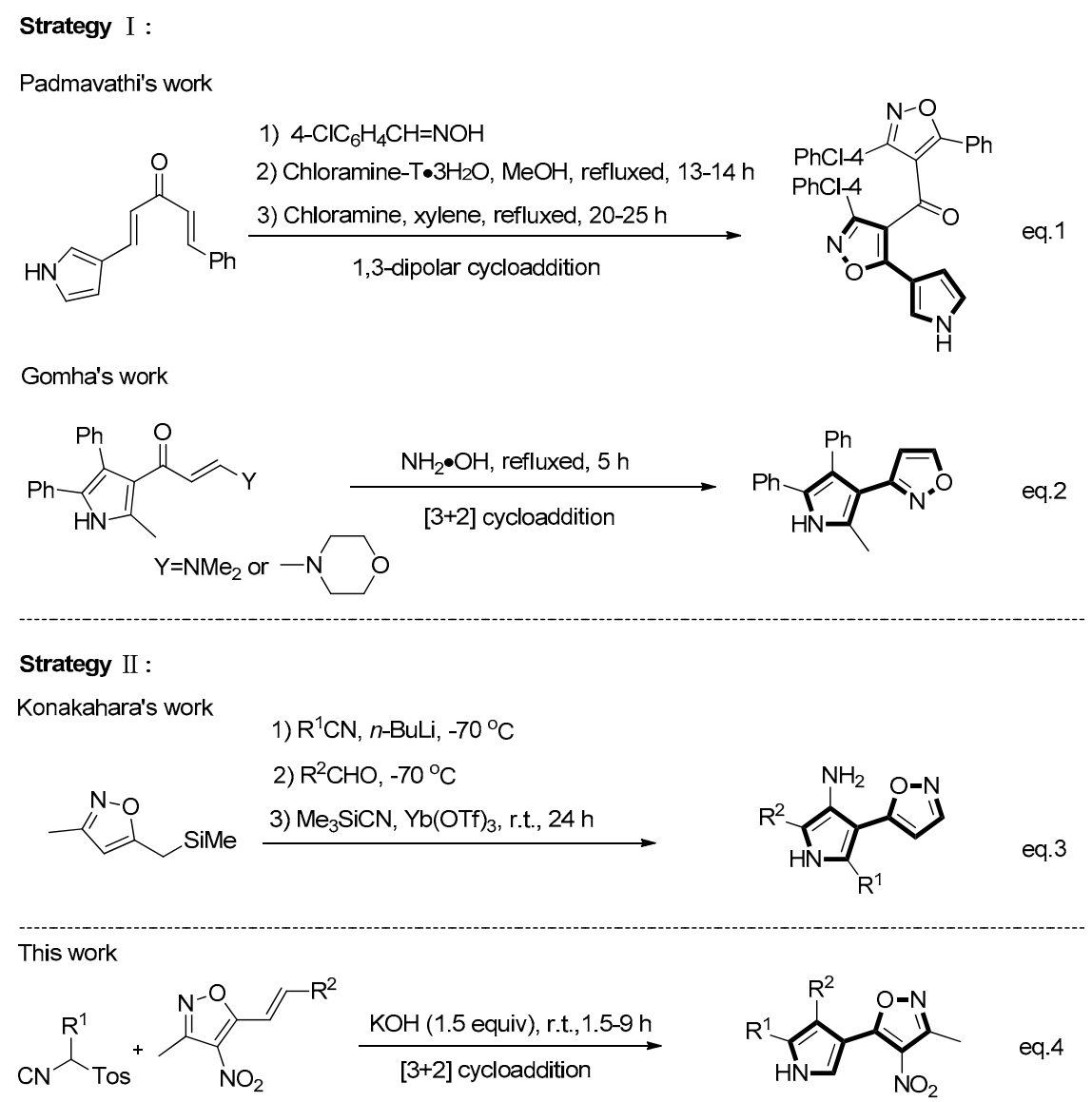

Scheme 1. Comparison between the selected existing literature examples and this work.

In the past decades, a variety of elegant methods for the synthesis of pyrroles or oligofunctional pyrroles have been reported, including the classical Hantzsch reaction [10], the Paal-Knorr cyclization reaction [10], the van Leusen cyclization [11], and other cyclizations [11]. Among them, the [3+2] cycloaddition of tosylmethyl isocyanide with electron-deficient olefins, developed by van Leusen et al., is one of the most promising methods [12-18]. A wide range of electron-deficient olefins, such as $\alpha, \beta$-unsaturated esters, ketones or nitriles, nitroolefins and styrenes, etc., are well tolerated in this reaction [19-36]. 3-Methyl-4-nitro-5-alkenylisoxazoles, developed by Adamo et al., are excellent activated olefins, which hold excellent potential for the generation of diversity [37-40]. In 2015, Adamo and co-workers reported an additional reaction of 3-methyl-4-nitro-5-alkenylisoxazoles and ethyl isocyanoacetate to give enantioenriched monoadducts; then, resulting adducts were subsequently 
cyclized to give 2,3-dihydropyrroles [41]. Although the stepwise synthesis of dihydropyrroles from styrylisoxazoles was developed [41], to our knowledge, the [3+2] cycloaddition reaction of styrylisoxazoles with TosMIC for the synthesis of isoxazolylpyrroles has not been reported so far. As part of our continued efforts to develop the heterocyclization of TosMIC [42-47], we report herein an expedient and convenient one-pot synthesis of isoxazole-substituted pyrrole derivatives from [3+2] cycloaddition of 3-methyl-4-nitro-5-styrylisoxazoles with TosMIC and analogs (Scheme 1, Equation (4)). Under basic conditions, various styrylisoxazoles reacted smoothly with TosMIC and analogs to deliver a wide range of polysubstituted isoxazolylpyrroles at ambient temperature.

\section{Results and Discussion}

Initially, the reaction of TosMIC 1a with (E)-5-(4-chlorostyryl)-3-methyl-4-nitroisoxazole $\mathbf{2} \mathbf{b}$ was tested for the optimization of the reaction conditions. It was found that the reaction of $\mathbf{1 a}$ and $\mathbf{2 b}$ to the formation of isoxazole substituted pyrrole $3 \mathbf{a b}$ in $84 \%$ yield (Table 1, entry 1 ) under DBU (1.5 equiv) in $\mathrm{CH} 3 \mathrm{CN}$ at room temperature for $1 \mathrm{~h}$. When the reaction time is prolonged to $6 \mathrm{~h}$ under the same conditions, the yield can be only improved to $87 \%$ (Table 1, entry 2). Decreasing (1.1 equiv) or increasing (1.5 equiv) the amount of TosMIC 1a lead to almost same yield ( $83 \%$ and $84 \%$ ) of $3 \mathbf{a b}$ (Table 1, entries 3 and 4). Among the screened bases such as DBU, $\mathrm{K}_{2} \mathrm{CO}_{3}, \mathrm{KOH}, \mathrm{TMG}, t$-BuOK and $\mathrm{NaOH}$ (Table 1, entries 4-9), $\mathrm{KOH}$ is optimal (Table 1, entry 6). Different solvents were also surveyed, with ethanol giving comparable yield of $3 a b$ (Table 1, entry 10). The [3+2]-cycloaddition reaction was slower, when the reaction was performed in DMF or THF (Table 1, entries 11 and 12).

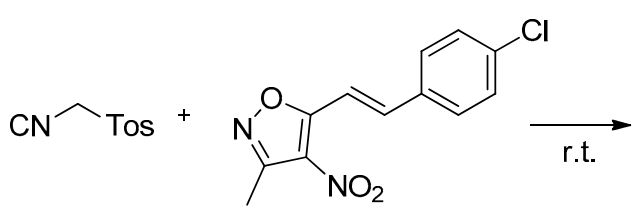

$1 \mathbf{a}$
$2 \mathbf{b}$

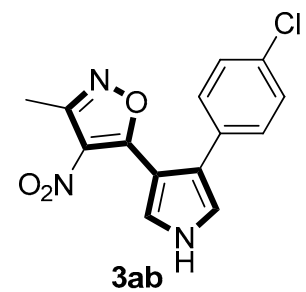

$3 a b^{H}$

Table 1. Optimization of the reaction conditions.

\begin{tabular}{cccccc}
\hline Entry & $\mathbf{1 a : 2 b}$ & Base (equiv) & Solvent & Time (h) & Yield (\%) $^{\text {a }}$ \\
\hline 1 & $1.3: 1$ & DBU (1.5) & $\mathrm{CH}_{3} \mathrm{CN}$ & 1.0 & 84 \\
2 & $1.3: 1$ & $\mathrm{DBU}(1.5)$ & $\mathrm{CH}_{3} \mathrm{CN}$ & 6.0 & 87 \\
3 & $1.1: 1$ & $\mathrm{DBU}(1.5)$ & $\mathrm{CH}_{3} \mathrm{CN}$ & 1.5 & 83 \\
4 & $1.5: 1$ & $\mathrm{DBU}(1.5)$ & $\mathrm{CH}_{3} \mathrm{CN}$ & 1.5 & 84 \\
5 & $1.3: 1$ & $\mathrm{~K}_{2} \mathrm{CO}_{3}(1.5)$ & $\mathrm{CH}_{3} \mathrm{CN}$ & 8.0 & 82 \\
$\mathbf{6}$ & $\mathbf{1 . 3 : 1}$ & $\mathrm{KOH}(1.5)$ & $\mathrm{CH}_{3} \mathrm{CN}$ & $\mathbf{2 . 5}$ & $\mathbf{9 0}$ \\
7 & $1.3: 1$ & $\mathrm{TMG} \mathrm{(1.5)}$ & $\mathrm{CH}_{3} \mathrm{CN}$ & 0.5 & 82 \\
8 & $1.3: 1$ & $t-\mathrm{BuOK}(1.5)$ & $\mathrm{CH}_{3} \mathrm{CN}$ & 1.5 & 77 \\
9 & $1.3: 1$ & $\mathrm{NaOH}(1.5)$ & $\mathrm{CH}_{3} \mathrm{CN}$ & 1.0 & 82 \\
10 & $1.3: 1$ & $\mathrm{KOH} \mathrm{(1.5)}$ & $\mathrm{EtOH}$ & 2.0 & 80 \\
11 & $1.3: 1$ & $\mathrm{KOH} \mathrm{(1.5)}$ & $\mathrm{DMF}$ & 1.5 & 63 \\
12 & $1.3: 1$ & $\mathrm{KOH} \mathrm{(1.5)}$ & THF & 2.0 & 70 \\
\hline \multicolumn{5}{c}{ a Yield of isolated product 3ab. }
\end{tabular}

With optimal conditions in hand (Table 1, entry 6), various (E)-3-methyl-4-nitro-5-styrylisoxazoles 2 were explored to investigate the generality of this tandem one-pot reaction for the synthesis of 3. The results are tabulated in Table 2. Substrates 2, with either electron-rich or electron-deficient aryl groups, afforded the double Michael adduct 3aa-al in excellent yields (Table 2, entries 1-10). Next, with the aim to explore the scope of the reaction mentioned above, a variety of (E)-3-methyl-4-nitro-5-(prop-1-en-1-yl)isoxazoles 2 were selected to react with TosMIC 1a under the optimized conditions. Further experiments showed that the reaction proceeded more efficiently for the $\mathbf{R}^{2}$ group on (E)-3-methyl-4-nitro-5-(prop-1-en-1-yl)isoxazoles 2, such as 2-furyl (2n), 2-thienyl (2o), 
2-naphthyl (2p), and styryl (2q) (these groups were well tolerated) (Table 2, entries 14-17). In general, a wide range of styrylisoxazoles 2 bearing various functional groups were reacted smoothly with TosMIC 1a under mild conditions, thus giving rise to the pyrrole products 3 in moderate to high yields.<smiles>[R]C=Cc1onc(C)c1[N+](=O)[O-]</smiles>

$1 \mathbf{a}$

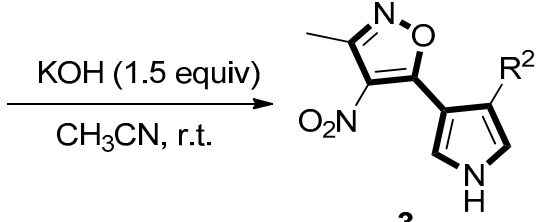

3

Table 2. Synthesis of 3-isoxazole bisubstituted pyrrole derivatives 1-17.

\begin{tabular}{|c|c|c|c|c|}
\hline Entry & $\mathbf{R}^{2}$ & Time (h) & 3 & Yield (\%) ${ }^{a}$ \\
\hline 1 & $\mathrm{Ph}$ & 4.0 & aa & 93 \\
\hline 2 & $4-\mathrm{ClC}_{6} \mathrm{H}_{4}$ & 2.5 & $\mathbf{a b}$ & 90 \\
\hline 3 & $4-\mathrm{BrC}_{6} \mathrm{H}_{4}$ & 5.5 & ac & 88 \\
\hline 4 & $4-\mathrm{NO}_{2} \mathrm{C}_{6} \mathrm{H}_{4}$ & 4.5 & ad & 90 \\
\hline 5 & $4-\mathrm{CH}_{3} \mathrm{C}_{6} \mathrm{H}_{4}$ & 3.5 & ae & 97 \\
\hline 6 & $3-\mathrm{CH}_{3} \mathrm{C}_{6} \mathrm{H}_{4}$ & 4.0 & af & 87 \\
\hline 7 & $3-\mathrm{OCH}_{3} \mathrm{C}_{6} \mathrm{H}_{4}$ & 1.5 & ag & 86 \\
\hline 8 & $3-\mathrm{ClC}_{6} \mathrm{H}_{4}$ & 3.5 & $\mathrm{ah}$ & 86 \\
\hline 9 & $2-\mathrm{CH}_{3} \mathrm{C}_{6} \mathrm{H}_{4}$ & 1.5 & ai & 92 \\
\hline 10 & $2-\mathrm{ClC}_{6} \mathrm{H}_{4}$ & 5.0 & aj & 89 \\
\hline 11 & $2,3-\mathrm{ClC}_{6} \mathrm{H}_{3}$ & 3.5 & ak & 57 \\
\hline 12 & $3,4-\mathrm{Cl}_{2} \mathrm{C}_{6} \mathrm{H}_{3}$ & 4.5 & al & 78 \\
\hline 13 & $2,5-\left(\mathrm{OCH}_{3}\right)_{2} \mathrm{C}_{6} \mathrm{H}_{3}$ & 4.0 & am & 86 \\
\hline 14 & 2-furyl & 3.5 & an & 84 \\
\hline 15 & 2-thienyl & 3.5 & ao & 81 \\
\hline 16 & 2-naphthyl & 5.0 & ap & 90 \\
\hline 17 & $\mathrm{C}_{6} \mathrm{H}_{5} \mathrm{CH}=\mathrm{CH}$ & 3.0 & aq & 82 \\
\hline
\end{tabular}

a Yields of isolated product.

To our delight, under optimal conditions (Table 1, entry 6), further experiments showed that the $\mathrm{R}^{1}$ group on TosMIC 1a, such as the ethyl (1b), allyl (1c), phenyl (1d), benzyl (1e), and p-methylbenzyl (1f) groups, also gave the corresponding trisubstituted pyrroles 3 in high yield (Table 3, entries 1-5). Therefore, a wide range of trisubstituted pyrrole derivatives were obtained under mild conditions. The configurations of pyrroles 3aa-fb were assigned by NMR and high-resolution mass spectra, and the structure of 3ac was further confirmed by the X-ray diffraction analysis (Figure 2).

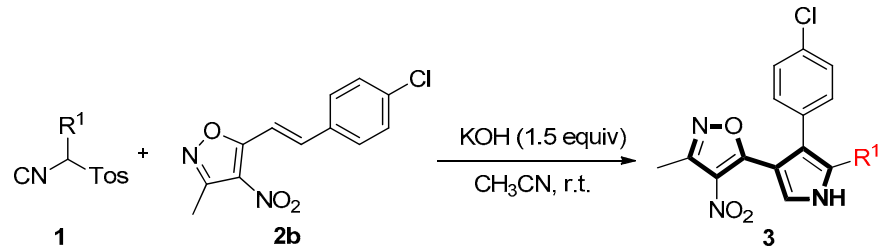

Table 3. Synthesis of 3-isoxazole trisubstituted pyrrole derivatives 1-5.

\begin{tabular}{ccccc}
\hline Entry & $\mathbf{R}^{\mathbf{1}}$ & Time (h) & $\mathbf{3}$ & Yield (\%) $^{\mathbf{a}}$ \\
\hline 1 & $\mathrm{CH}_{3} \mathrm{CH}_{2}$ & 8.0 & $\mathbf{b b}$ & 67 \\
2 & allyl & 9.0 & $\mathbf{c b}$ & 56 \\
3 & $\mathrm{C}_{6} \mathrm{H}_{5}$ & 4.0 & $\mathbf{d b}$ & 81 \\
4 & $\mathrm{C}_{6} \mathrm{H}_{5} \mathrm{CH}_{2}$ & 7.0 & $\mathbf{e b}$ & 78 \\
5 & $4-\mathrm{CH}_{3} \mathrm{C}_{6} \mathrm{H}_{4} \mathrm{CH}_{2}$ & 5.0 & $\mathbf{f b}$ & 83 \\
\hline \multicolumn{5}{c}{ a Yields of isolated product. }
\end{tabular}

${ }^{a}$ Yields of isolated product. 


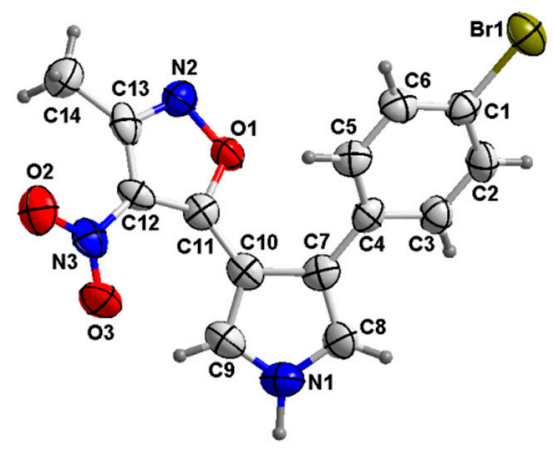

Figure 2. ORTEP drawing of 3ac.

Generally, a stepwise mechanism rather than a concerted process is proposed in the van Leusen pyrrole synthesis from the [3+2] cycloaddition of electron-deficient olefins with TosMIC [19-36]. Thus, on the basis of the related reports [43-48] and above-stated results, a possible mechanism for the synthesis of 3 was proposed and depicted in Scheme 2. First, addition of TosMIC $\mathbf{1}$ to (E)-3-methyl-4-nitro-5-(prop-1-en-1-yl)isoxazole 2, in the presence of $\mathrm{KOH}$ in $\mathrm{CH}_{3} \mathrm{CN}$, leads to the adduct (A). Intramolecular cyclization of the adduct (A) occurs to produce the intermediate (B) [47]. Then, protontropic shifts, followed by the elimination of a toluenesulfinate anion to produce the intermediate (E) and the final hydrogen shift, deliver the 3-isoxazole-substituted pyrrole derivatives 3 .

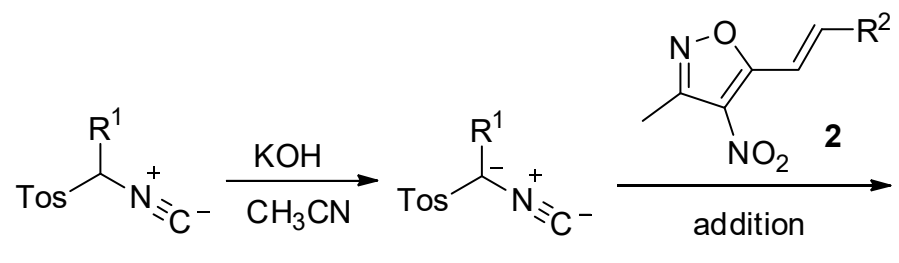

1

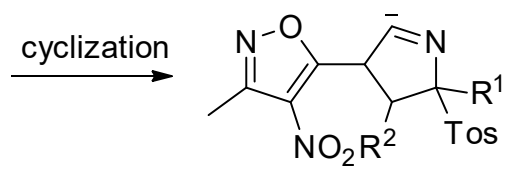

B<smiles>[R2]C1C(c2onc(C)c2[N+](=O)[O-])C=NC1([Y8])[Z7]([H])([H])CC</smiles>

C<smiles>[R]C(Sc1onc(C)c1[N+](=O)[O-])C([Y])(N=CC)C([R])([Y])CC</smiles>

A<smiles>[R2]C1C(c2onc(C)c2[N+](=O)[O-])=C[N-]C1([R7])[Z19]</smiles>

D<smiles>[R]C1=C([R2])C(c2onc(C)c2[N+](=O)[O-])C=N1</smiles>

$\mathrm{E}$<smiles>[R]c1[nH]cc(-c2onc(C)c2[N+](=O)[O-])c1[R2]</smiles>

3

Scheme 2. Proposed mechanism for the formation of 3.

\section{Experimental}

\subsection{General}

All reagents were commercial and used without further purification, unless otherwise indicated. Chromatography was carried on flash silica gel (300-400 mesh). All reactions were monitored by TLC, which was performed on precoated aluminum sheets of silica gel 60 (F254). Melting points were uncorrected. The ${ }^{1} \mathrm{H}-\mathrm{NMR}$ and ${ }^{13} \mathrm{C}-\mathrm{NMR}$ spectra were determined at $25^{\circ} \mathrm{C}$ at $600 \mathrm{MHz}, 150 \mathrm{MHz}$, or $125 \mathrm{MHz}$, respectively, with TMS as an internal standard. All shifts are given in ppm. High-resolution 
mass spectra (HRMS) were obtained using a Bruker microTOF II focus spectrometer (ESI). Crystal data was obtained by a Bruker SMART X-Ray single crystal diffractometer (Bruker, Germany). The substrates (E)-3-methyl-4-nitro-5-styrylisoxazoles 2 were prepared by a similar method as reported papers [49,50]. More informations can be found in the supplementary materials.

\subsection{Synthesis of $\mathbf{3 a a}-\mathbf{3} \mathbf{f b}$}

General procedures for the synthesis of 3 (taking $3 \mathrm{ab}$ as an example): to the mixture of tosylmethyl isocyanide $\mathbf{1 a}(50.7 \mathrm{mg}, 0.26 \mathrm{mmol}$ ) and (E)-5-(4-chlorostyryl)-3-methyl-4-nitroisoxazole $\mathbf{2 b}$ (52.8 $\mathrm{mg}$, $0.2 \mathrm{mmol})$ in $\mathrm{CH}_{3} \mathrm{CN}(2 \mathrm{~mL})$ was added $\mathrm{KOH}(16.8 \mathrm{mg}, 0.3 \mathrm{mmol})$, in one portion, at room temperature. The reaction mixture was stirred and monitored by TLC. After the substrate $\mathbf{2} \mathbf{b}$ was consumed, the solvent was removed under vacuum. The crude product was subjected to column chromatography on silica gel (petroleum ether $/ \mathrm{EtOAc}=8: 1)$ to give $3 \mathbf{a b}(54.5 \mathrm{mg}, 90 \%)$ as a green solid.

3-Methyl-4-nitro-5-(4-phenyl-1H-pyrrol-3-yl)isoxazole (3aa). Green solid, yield 93\%, m.p. $174-176{ }^{\circ} \mathrm{C}$. ${ }^{1} \mathrm{H}-\mathrm{NMR}\left(\mathrm{DMSO}-d_{6}, 600 \mathrm{MHz}\right) \delta 2.47(\mathrm{~s}, 3 \mathrm{H}), 7.16(\mathrm{~s}, 1 \mathrm{H}), 7.21(\mathrm{t}, J=6 \mathrm{~Hz}, 3 \mathrm{H}), 7.29(\mathrm{t}, J=7.8 \mathrm{~Hz}, 2 \mathrm{H})$, $7.81(\mathrm{~s}, 1 \mathrm{H}), 11.96(\mathrm{~s}, 1 \mathrm{H}) .{ }^{13} \mathrm{C}-\mathrm{NMR}$ (DMSO- $\left.d_{6}, 150 \mathrm{MHz}\right) \delta 12.2,105.7,119.8,125.4,126.5,126.7,127.6$, $128.1,128.8,135.4,156.5,167.0$. HRMS (ESI-TOF) $m / z$ : Calcd. for $\mathrm{C}_{14} \mathrm{H}_{12} \mathrm{~N}_{3} \mathrm{O}_{3}{ }^{+}\left([\mathrm{M}+\mathrm{H}]^{+}\right) 270.0873$. Found: 270.0865 .

5-(4-(4-Chlorophenyl)-1H-pyrrol-3-yl)-3-methyl-4-nitroisoxazole (3ab). Green solid, yield 90\%, m.p. 183-185 ${ }^{\circ} \mathrm{C} .{ }^{1} \mathrm{H}-\mathrm{NMR}\left(\mathrm{DMSO}-d_{6}, 600 \mathrm{MHz}\right) \delta 2.47(\mathrm{~s}, 3 \mathrm{H}), 7.20(\mathrm{~s}, 1 \mathrm{H}), 7.23(\mathrm{~d}, J=8.4 \mathrm{~Hz}, 2 \mathrm{H})$, $7.34(\mathrm{~d}, J=8.4 \mathrm{~Hz}, 2 \mathrm{H}), 7.81(\mathrm{~s}, 1 \mathrm{H}), 12.00(\mathrm{~s}, 1 \mathrm{H}) .{ }^{13} \mathrm{C}-\mathrm{NMR}$ (DMSO-d $\left.d_{6}, 150 \mathrm{MHz}\right), \delta$ 12.2, 105.7, $120.2,124.1,126.8,127.7,128.8,129.8,131.4,134.4,156.6,166.7$. HRMS (ESI-TOF) $m / z$ : Calcd. for $\mathrm{C}_{14} \mathrm{H}_{11} \mathrm{ClN}_{3} \mathrm{O}_{3}{ }^{+}\left([\mathrm{M}+\mathrm{H}]^{+}\right)$304.0483. Found: 304.0477.

5-(4-(4-Bromophenyl)-1H-pyrrol-3-yl)-3-methyl-4-nitroisoxazole (3ac). Green solid, yield 88\%, m.p. 191-193 ${ }^{\circ} \mathrm{C} .{ }^{1} \mathrm{H}-\mathrm{NMR}$ (DMSO- $\left.d_{6}, 600 \mathrm{MHz}\right) \delta 2.48(\mathrm{~s}, 3 \mathrm{H}), 7.17(\mathrm{~d}, J=8.0 \mathrm{~Hz}, 2 \mathrm{H}), 7.21(\mathrm{~s}, 1 \mathrm{H})$, $7.48(\mathrm{~d}, J=8.0 \mathrm{~Hz}, 2 \mathrm{H}), 7.81(\mathrm{~s}, 1 \mathrm{H}), 12.01(\mathrm{~s}, 1 \mathrm{H}) .{ }^{13} \mathrm{C}-\mathrm{NMR}$ (DMSO-d $\left.d_{6}, 150 \mathrm{MHz}\right) \delta 12.2,105.6$, 119.8, 120.1, 124.0, 126.6, 127.6, 130.0, 131.6, 134.8, 156.4, 166.6. HRMS (ESI-TOF) $m / z$ : Calcd. for $\mathrm{C}_{14} \mathrm{H}_{11} \mathrm{BrN}_{3} \mathrm{O}_{3}{ }^{+}\left([\mathrm{M}+\mathrm{H}]^{+}\right)$347.9978. Found: 347.9978.

3-Methyl-4-nitro-5-(4-(4-nitrophenyl)-1H-pyrrol-3-yl)isoxazole (3ad). Green solid, yield 90\%, m.p. 183-185 ${ }^{\circ} \mathrm{C} .{ }^{1} \mathrm{H}-\mathrm{NMR}\left(\mathrm{DMSO}-d_{6}, 600 \mathrm{MHz}\right) \delta 2.48(\mathrm{~s}, 3 \mathrm{H}), 7.42(\mathrm{~s}, 1 \mathrm{H}), 7.49(\mathrm{~d}, J=9 \mathrm{~Hz}, 2 \mathrm{H}), 7.85$ $(\mathrm{s}, 1 \mathrm{H}), 8.14(\mathrm{~d}, J=9 \mathrm{~Hz}, 2 \mathrm{H}), 12.20(\mathrm{~s}, 1 \mathrm{H}) .{ }^{13} \mathrm{C}-\mathrm{NMR}$ (DMSO- $\left.d_{6}, 150 \mathrm{MHz}\right) \delta 12.2,105.9,121.8,123.2$, 124.2, 127.3, 128.0, 128.6, 142.6, 146.0, 156.7, 166.4. HRMS (ESI-TOF) $m / z$ : Calcd. for $\mathrm{C}_{14} \mathrm{H}_{11} \mathrm{~N}_{4} \mathrm{O}_{5}{ }^{+}$ $\left([\mathrm{M}+\mathrm{H}]^{+}\right)$315.0724. Found: 315.0726.

3-Methyl-4-nitro-5-(4-(p-tolyl)-1H-pyrrol-3-yl)isoxazole (3ae). Yellow solid, yield 97\%, m.p. 157-159 ${ }^{\circ} \mathrm{C}$. ${ }^{1} \mathrm{H}-\mathrm{NMR}\left(\mathrm{CDCl}_{3}, 600 \mathrm{MHz}\right) \delta 2.34(\mathrm{~s}, 3 \mathrm{H}), 2.57(\mathrm{~s}, 3 \mathrm{H}), 6.88(\mathrm{t}, J=2.4 \mathrm{~Hz}, 1 \mathrm{H}), 7.13-7.16(\mathrm{~m}, 4 \mathrm{H})$, $7.84\left(\mathrm{dd}, J_{1}=2.4 \mathrm{~Hz}, J_{2}=0.6 \mathrm{~Hz}, 1 \mathrm{H}\right), 8.99(\mathrm{~s}, 1 \mathrm{H}) .{ }^{13} \mathrm{C}-\mathrm{NMR}\left(\mathrm{CDCl}_{3}, 125 \mathrm{MHz}\right) \delta 12.0,21.1,106.8$, $118.3,125.4,126.4,127.4,128.1,129.0,131.4,136.5,156.0,166.5$. HRMS (ESI-TOF) $m / z$ : Calcd. for $\mathrm{C}_{15} \mathrm{H}_{13} \mathrm{~N}_{3} \mathrm{NaO}_{3}{ }^{+}\left([\mathrm{M}+\mathrm{Na}]^{+}\right)$306.0849. Found: 306.0846.

3-Methyl-4-nitro-5-(4-(m-tolyl)-1H-pyrrol-3-yl)isoxazole (3af). Green solid, yield 87\%, m.p. $168-170{ }^{\circ} \mathrm{C}$. ${ }^{1} \mathrm{H}-\mathrm{NMR}$ (DMSO- $\left.d_{6}, 600 \mathrm{MHz}\right) \delta 2.27(\mathrm{~s}, 3 \mathrm{H}), 2.47(\mathrm{~s}, 3 \mathrm{H}), 6.96(\mathrm{~d}, J=7.8 \mathrm{~Hz}, 1 \mathrm{H}), 7.03(\mathrm{~d}, J=7.8 \mathrm{~Hz}$, $1 \mathrm{H}), 7.08(\mathrm{~s}, 1 \mathrm{H}), 7.14(\mathrm{t}, J=2.4 \mathrm{~Hz}, 1 \mathrm{H}), 7.16(\mathrm{t}, J=7.8 \mathrm{~Hz}, 1 \mathrm{H}), 7.80(\mathrm{t}, J=2.4 \mathrm{~Hz}, 1 \mathrm{H}), 11.95(\mathrm{~s}, 1 \mathrm{H})$. ${ }^{13} \mathrm{C}-\mathrm{NMR}\left(\mathrm{DMSO}-d_{6}, 150 \mathrm{MHz}\right) \delta 12.2,21.6,105.7,119.7,125.2,125.4,126.4,127.4,127.6,128.6,128.7$, 135.3, 137.8, 156.4, 167.0. HRMS (ESI-TOF) $m / z$ : Calcd. for $\mathrm{C}_{15} \mathrm{H}_{14} \mathrm{~N}_{3} \mathrm{O}_{3}{ }^{+}\left([\mathrm{M}+\mathrm{H}]^{+}\right)$284.1030. Found: 284.1035 .

5-(4-(3-Methoxyphenyl)-1H-pyrrol-3-yl)-3-methyl-4-nitroisoxazole (3ag). Yellow solid, yield 86\%, m.p. 169-171 ${ }^{\circ} \mathrm{C} .{ }^{1} \mathrm{H}-\mathrm{NMR}$ (DMSO- $\left.d_{6}, 600 \mathrm{MHz}\right) \delta 2.47(\mathrm{~s}, 3 \mathrm{H}), 3.70(\mathrm{~s}, 3 \mathrm{H}), 6.75-6.79(\mathrm{~m}, 3 \mathrm{H}), 7.18-7.20$ $(\mathrm{m}, 2 \mathrm{H}), 7.77-7.78(\mathrm{~m}, 1 \mathrm{H}), 11.95$ (s, $1 \mathrm{H}) .{ }^{13} \mathrm{C}-\mathrm{NMR}$ (DMSO- $\left.d_{6}, 150 \mathrm{MHz}\right) \delta 12.2,55.5,105.7,112.3$, 
113.5, 119.9, 120.4, 125.2, 126.4, 127.7, 129.9, 136.7, 156.5, 159.7, 167.0. HRMS (ESI-TOF) $m / z$ : Calcd. for $\mathrm{C}_{15} \mathrm{H}_{13} \mathrm{~N}_{3} \mathrm{NaO}_{4}^{+}\left([\mathrm{M}+\mathrm{Na}]^{+}\right)$322.0798. Found: 322.0795 .

5-(4-(3-Chlorophenyl)-1H-pyrrol-3-yl)-3-methyl-4-nitroisoxazole (3ah). Paleyellow solid, yield 86\%, m.p.163-165 ${ }^{\circ} \mathrm{C} .{ }^{1} \mathrm{H}-\mathrm{NMR}$ (DMSO-d $\left.6,600 \mathrm{MHz}\right) \delta 2.47$ (s, 3H), 7.13 (d, J = $\left.7.8 \mathrm{~Hz}, 1 \mathrm{H}\right), 7.26-7.32$ $(\mathrm{m}, 4 \mathrm{H}), 7.83(\mathrm{t}, J=2.4 \mathrm{~Hz}, 1 \mathrm{H}), 12.05(\mathrm{~s}, 1 \mathrm{H}) .{ }^{13} \mathrm{C}-\mathrm{NMR}\left(\mathrm{DMSO}-d_{6}, 150 \mathrm{MHz}\right) \delta 12.2,105.7,120.6$, $123.7,126.5,126.7,126.8,127.6,127.7,130.6,133.5,137.6,156.5,166.6$. HRMS (ESI-TOF) $m / z$ : Calcd. for $\mathrm{C}_{14} \mathrm{H}_{11} \mathrm{ClN}_{3} \mathrm{O}_{3}{ }^{+}\left([\mathrm{M}+\mathrm{H}]^{+}\right)$304.0483. Found: 304.0474.

3-Methyl-4-nitro-5-(4-(o-tolyl)-1H-pyrrol-3-yl)isoxazole (3ai). Yellow solid, yield 92\%, m.p. 185-187 ${ }^{\circ} \mathrm{C}$. ${ }^{1} \mathrm{H}-\mathrm{NMR}\left(\mathrm{CDCl}_{3}, 600 \mathrm{MHz}\right) \delta 2.11(\mathrm{~s}, 3 \mathrm{H}), 2.52(\mathrm{~s}, 3 \mathrm{H}), 6.79-6.80(\mathrm{~m}, 1 \mathrm{H}), 7.16-7.17(\mathrm{~m}, 2 \mathrm{H}), 7.21-7.24$ $(\mathrm{m}, 2 \mathrm{H}), 8.11-8.12(\mathrm{~m}, 1 \mathrm{H}), 8.95(\mathrm{~s}, 1 \mathrm{H}) .{ }^{13} \mathrm{C}-\mathrm{NMR}\left(\mathrm{CDCl}_{3}, 125 \mathrm{MHz}\right) \delta 12.1,20.1,108.5,118.9,125.4$, $125.4,125.4,126.7,127.5,129.9,130.4,134.2,136.9,155.9,166.2$. HRMS (ESI-TOF) $m / z$ : Calcd. for $\mathrm{C}_{15} \mathrm{H}_{13} \mathrm{~N}_{3} \mathrm{NaO}_{3}{ }^{+}\left([\mathrm{M}+\mathrm{Na}]^{+}\right)$306.0849. Found: 306.0854.

5-(4-(2-Chlorophenyl)-1H-pyrrol-3-yl)-3-methyl-4-nitroisoxazole (3aj). Green solid, yield 89\%, m.p. 165-167 ${ }^{\circ} \mathrm{C} .{ }^{1} \mathrm{H}-\mathrm{NMR}\left(\mathrm{CDCl}_{3}, 600 \mathrm{MHz}\right) \delta 2.54(\mathrm{~s}, 3 \mathrm{H}), 6.90(\mathrm{t}, J=2.4 \mathrm{~Hz}, 1 \mathrm{H}), 7.24-7.27(\mathrm{~m}, 2 \mathrm{H})$, $7.31\left(\mathrm{dd}, J_{1}=3.6 \mathrm{~Hz}, J_{2}=2.4 \mathrm{~Hz}, 1 \mathrm{H}\right), 7.4\left(\mathrm{dd}, J_{1}=3.6 \mathrm{~Hz}, J_{2}=2.4 \mathrm{~Hz}, 1 \mathrm{H}\right), 8.11\left(\mathrm{dd}, J_{1}=2.4 \mathrm{~Hz}\right.$, $\left.J_{2}=0.6 \mathrm{~Hz}, 1 \mathrm{H}\right), 8.98(\mathrm{~s}, 1 \mathrm{H}) .{ }^{13} \mathrm{C}-\mathrm{NMR}\left(\mathrm{CDCl}_{3}, 125 \mathrm{MHz}\right) \delta 12.1,108.6,119.6,123.3,125.4,126.6,128.7$, $129.5,131.6,133.6,133.9,156.0,166.1$. HRMS (ESI-TOF) $m / z$ : Calcd. for $\mathrm{C}_{14} \mathrm{H}_{11} \mathrm{ClN}_{3} \mathrm{O}_{3}{ }^{+}\left([\mathrm{M}+\mathrm{H}]^{+}\right)$ 304.0483. Found: 304.0482 .

5-(4-(2,3-Dichlorophenyl)-1H-pyrrol-3-yl)-3-methyl-4-nitroisoxazole (3ak). Green solid, yield 57\%, m.p. 177-179 ${ }^{\circ} \mathrm{C} .{ }^{1} \mathrm{H}-\mathrm{NMR}\left(\mathrm{CDCl}_{3}, 600 \mathrm{MHz}\right) \delta 2.54(\mathrm{~s}, 3 \mathrm{H}), 6.92(\mathrm{~s}, 1 \mathrm{H}), 7.20(\mathrm{~d}, \mathrm{~J}=8.8 \mathrm{~Hz}, 2 \mathrm{H}), 7.43$ $(\mathrm{d}, J=8.8 \mathrm{~Hz}, 1 \mathrm{H}), 8.15(\mathrm{~s}, 1 \mathrm{H}), 8.95(\mathrm{~s}, 1 \mathrm{H}) .{ }^{13} \mathrm{C}-\mathrm{NMR}\left(\mathrm{CDCl}_{3}, 125 \mathrm{MHz}\right) \delta 12.1,108.7,119.7,123.2,125.5$, 126.9, 129.7, 129.9, 132.5, 133.3, 136.0, 156.0, 165.7. HRMS (ESI-TOF) $m / z$ : Calcd. for $\mathrm{C}_{14} \mathrm{H}_{10} \mathrm{Cl}_{2} \mathrm{~N}_{3} \mathrm{O}_{3}{ }^{+}$ $\left([\mathrm{M}+\mathrm{H}]^{+}\right)$338.0094. Found: 338.0080 .

5-(4-(3,4-Dichlorophenyl)-1H-pyrrol-3-yl)-3-methyl-4-nitroisoxazole (3al). Green solid, yield 78\%, m.p. 174-176 ${ }^{\circ} \mathrm{C} .{ }^{1} \mathrm{H}-\mathrm{NMR}\left(\mathrm{CDCl}_{3}, 600 \mathrm{MHz}\right) \delta 2.58(\mathrm{~s}, 3 \mathrm{H}), 6.95(\mathrm{t}, J=2.4 \mathrm{~Hz}, 1 \mathrm{H}), 7.06\left(\mathrm{dd}, J_{1}=1.8 \mathrm{~Hz}\right.$, $\left.J_{2}=6.6 \mathrm{~Hz}, 1 \mathrm{H}\right), 7.38-7.39(\mathrm{~m}, 2 \mathrm{H}), 7.94-7.95(\mathrm{~m}, 1 \mathrm{H}), 8.92(\mathrm{~s}, 1 \mathrm{H}) .{ }^{13} \mathrm{C}-\mathrm{NMR}\left(\mathrm{CDCl}_{3}, 125 \mathrm{MHz}\right) \delta 12.1$, 107.1, 118.9, 124.3, 125.8, 127.8, 130.1, 130.2, 131.0, 132.3, 134.5, 156.2, 165.6. HRMS (ESI-TOF) $m / z$ : Calcd. for $\mathrm{C}_{14} \mathrm{H}_{10} \mathrm{Cl}_{2} \mathrm{~N}_{3} \mathrm{O}_{3}{ }^{+}\left([\mathrm{M}+\mathrm{H}]^{+}\right)$338.0094. Found: 338.0080.

5-(4-(2,5-Dimethoxyphenyl)-1H-pyrrol-3-yl)-3-methyl-4-nitroisoxazole (3am). Yellow solid, yield 86\%, m.p. 172-174 ${ }^{\circ} \mathrm{C} .{ }^{1} \mathrm{H}-\mathrm{NMR}\left(\mathrm{DMSO}-d_{6}, 600 \mathrm{MHz}\right) \delta 2.46(\mathrm{~s}, 3 \mathrm{H}), 3.33(\mathrm{~s}, 3 \mathrm{H}), 3.71(\mathrm{~s}, 3 \mathrm{H}), 6.78-6.80(\mathrm{~m}, 1 \mathrm{H})$, 6.82-6.84 (m, 2H), $7.08(\mathrm{t}, J=2.4 \mathrm{~Hz}, 1 \mathrm{H}), 7.80(\mathrm{t}, J=3 \mathrm{~Hz}, 1 \mathrm{H}), 11.89(\mathrm{~s}, 1 \mathrm{H}) .{ }^{13} \mathrm{C}-\mathrm{NMR}\left(\mathrm{DMSO}-d_{6}\right.$, $150 \mathrm{MHz}) \delta 12.1,55.7,55.8,107.3,112.4,112.8,116.3,120.4,121.6,125.2,125.7,126.6,150.5,153.5,156.0$, 168.0. HRMS (ESI-TOF) $m / z$ : Calcd. for $\mathrm{C}_{16} \mathrm{H}_{16} \mathrm{~N}_{3} \mathrm{O}_{5}{ }^{+}\left([\mathrm{M}+\mathrm{H}]^{+}\right)$330.1084. Found: 330.1095.

5-(4-(Furan-2-yl)-1H-pyrrol-3-yl)-3-methyl-4-nitroisoxazole (3an). Yellow solid, yield 84\%, m.p. 148-150 ${ }^{\circ} \mathrm{C} .{ }^{1} \mathrm{H}-\mathrm{NMR}\left(\mathrm{DMSO}-d_{6}, 600 \mathrm{MHz}\right) \delta 2.51(\mathrm{~s}, 3 \mathrm{H}), 6.34(\mathrm{~d}, J=3 \mathrm{~Hz}, 1 \mathrm{H}), 6.45\left(\mathrm{dd}, J_{1}=1.8 \mathrm{~Hz}\right.$, $\left.J_{2}=1.2 \mathrm{~Hz}, 1 \mathrm{H}\right), 7.31(\mathrm{~d}, J=1.8 \mathrm{~Hz}, 1 \mathrm{H}), 7.52(\mathrm{~s}, 1 \mathrm{H}), 7.73(\mathrm{~s}, 1 \mathrm{H}), 12.02(\mathrm{~s}, 1 \mathrm{H}) .{ }^{13} \mathrm{C}-\mathrm{NMR}\left(\mathrm{DMSO}-d_{6}\right.$, $150 \mathrm{MHz}) \delta 12.1,104.8,105.5,111.8,115.1,119.5,125.9,128.0,141.9,149.3,156.5,166.5$. HRMS (ESI-TOF) $m / z$ : Calcd. for $\mathrm{C}_{12} \mathrm{H}_{10} \mathrm{~N}_{3} \mathrm{O}_{4}{ }^{+}\left([\mathrm{M}+\mathrm{H}]^{+}\right)$260.0666. Found: 260.0669 .

3-Methyl-4-nitro-5-(4-(thiophen-2-yl)-1H-pyrrol-3-yl)isoxazole (3ao). Yellow solid, yield 81\%, m.p. 115-117 ${ }^{\circ} \mathrm{C} .{ }^{1} \mathrm{H}-\mathrm{NMR}\left(\mathrm{DMSO}-d_{6}, 600 \mathrm{MHz}\right) \delta 2.49$ (s, 3H), 6.90 (d, J=3 Hz, 1H), 6.99 (dd, J = 3.6 Hz, $J=1.2 \mathrm{~Hz}, 1 \mathrm{H}), 7.21(\mathrm{t}, J=2.4 \mathrm{~Hz}, 1 \mathrm{H}), 7.37(\mathrm{~d}, J=5.4 \mathrm{~Hz}, 1 \mathrm{H}), 7.76(\mathrm{t}, J=2.4 \mathrm{~Hz}, 1 \mathrm{H}), 12.01(\mathrm{~s}, 1 \mathrm{H})$. ${ }^{13}$ C-NMR (DMSO- $\left.d_{6}, 150 \mathrm{MHz}\right) \delta 12.1,105.8,117.9,120.2,124.8,124.9,126.2,128.0,128.0,136.6,156.5$, 166.5. HRMS (ESI-TOF) $m / z$ : Calcd. for $\mathrm{C}_{12} \mathrm{H}_{10} \mathrm{~N}_{3} \mathrm{O}_{3} \mathrm{~S}^{+}\left([\mathrm{M}+\mathrm{H}]^{+}\right)$276.0437. Found: 276.0446.

3-Methyl-5-(4-(naphthalen-2-yl)-1H-pyrrol-3-yl)-4-nitroisoxazole (3ap). Yellow solid, yield 90\%, m.p. 210-212 ${ }^{\circ} \mathrm{C} .{ }^{1} \mathrm{H}-\mathrm{NMR}$ (DMSO-d, $\left.600 \mathrm{MHz}\right) \delta 2.49(\mathrm{~s}, 3 \mathrm{H}), 7.32(\mathrm{~s}, 1 \mathrm{H}), 7.39(\mathrm{~d}, J=8.4 \mathrm{~Hz}, 1 \mathrm{H})$, 7.46-7.48 (m, 2H), 7.79 (s, 1H), $7.84(\mathrm{~d}, J=7.2 \mathrm{~Hz}, 2 \mathrm{H}), 7.88(\mathrm{~d}, J=7.8 \mathrm{~Hz}, 1 \mathrm{H}), 7.90(\mathrm{~s}, 1 \mathrm{H}), 12.07$ (s, $1 \mathrm{H})$. 
${ }^{13}$ C-NMR (DMSO- $\left.d_{6}, 150 \mathrm{MHz}\right) \delta 12.2,105.9,120.3,125.3,125.8,126.0,126.6,126.7,127.2,127.6,127.9$, 128.1, 128.2, 132.1, 133.0, 133.7, 156.5, 166.9. HRMS (ESI-TOF) $m / z$ : Calcd. for $\mathrm{C}_{18} \mathrm{H}_{14} \mathrm{~N}_{3} \mathrm{O}_{3}{ }^{+}\left([\mathrm{M}+\mathrm{H}]^{+}\right)$ 320.1030. Found: 320.1027.

(E)-3-Methyl-4-nitro-5-(4-styryl-1H-pyrrol-3-yl)isoxazole (3aq). Orange solid, yield 82\%, m.p. 177-179 ${ }^{\circ} \mathrm{C}$. ${ }^{1} \mathrm{H}-\mathrm{NMR}\left(\mathrm{CDCl}_{3}, 600 \mathrm{MHz}\right) \delta 2.62(\mathrm{~s}, 3 \mathrm{H}), 6.87(\mathrm{~d}, J=16.2 \mathrm{~Hz}, 1 \mathrm{H}), 7.17(\mathrm{~s}, 1 \mathrm{H}), 7.24(\mathrm{t}, J=7.2 \mathrm{~Hz}, 1 \mathrm{H})$, $7.33(\mathrm{~m}, 2 \mathrm{H}), 7.41(\mathrm{~d}, J=16.2 \mathrm{~Hz}, 1 \mathrm{H}), 7.47(\mathrm{~d}, J=7.8 \mathrm{~Hz}, 2 \mathrm{H}), 8.10-8.11(\mathrm{~m}, 1 \mathrm{H}), 8.83(\mathrm{~s}, 1 \mathrm{H}) .{ }^{13} \mathrm{C}-\mathrm{NMR}$ $\left(\mathrm{CDCl}_{3}, 125 \mathrm{MHz}\right) \delta 12.2,107.6,116.5,120.8,124.0,126.1,126.3,127.4,128.6,128.9,137.4,156.3,166.4$. HRMS (ESI-TOF) $m / z$ : Calcd. for $\mathrm{C}_{16} \mathrm{H}_{14} \mathrm{~N}_{3} \mathrm{O}_{3}{ }^{+}\left([\mathrm{M}+\mathrm{H}]^{+}\right)$296.1030. Found: 296.1028.

5-(4-(4-Chlorophenyl)-5-ethyl-1H-pyrrol-3-yl)-3-methyl-4-nitroisoxazole (3bb). Green solid, yield 67\%, m.p. 182-184 ${ }^{\circ} \mathrm{C} .{ }^{1} \mathrm{H}-\mathrm{NMR}\left(\mathrm{CDCl}_{3}, 600 \mathrm{MHz}\right) \delta 1.19(\mathrm{t}, J=7.8 \mathrm{~Hz}, 3 \mathrm{H}), 2.52(\mathrm{~s}, 3 \mathrm{H}), 2.60\left(\mathrm{dd}, J_{1}=7.8 \mathrm{~Hz}\right.$, $\left.J_{2}=7.2 \mathrm{~Hz}, 2 \mathrm{H}\right), 7.13(\mathrm{~m}, 2 \mathrm{H}), 7.32(\mathrm{~m}, 2 \mathrm{H}), 7.94(\mathrm{~d}, J=1.8 \mathrm{~Hz}, 1 \mathrm{H}), 8.66(\mathrm{~s}, 1 \mathrm{H}) .{ }^{13} \mathrm{C}-\mathrm{NMR}\left(\mathrm{CDCl}_{3}\right.$, $125 \mathrm{MHz}) \delta 12.1,14.1,18.9,29.7,108.1,120.3,123.9,128.3,131.2,132.8,133.2,133.4,156.0,166.1$. HRMS (ESI-TOF) $m / z$ : Calcd. for $\mathrm{C}_{16} \mathrm{H}_{15} \mathrm{ClN}_{3} \mathrm{O}_{3}{ }^{+}\left([\mathrm{M}+\mathrm{H}]^{+}\right)$332.0796. Found: 332.0799.

5-(5-Allyl-4-(4-chlorophenyl)-1H-pyrrol-3-yl)-3-methyl-4-nitroisoxazole (3cb). Green solid, yield 56\%, m.p. 171-173 ${ }^{\circ} \mathrm{C} .{ }^{1} \mathrm{H}-\mathrm{NMR}\left(\mathrm{CDCl}_{3}, 600 \mathrm{MHz}\right) \delta 2.52(\mathrm{~d}, 3 \mathrm{H}), 3.33(\mathrm{~d}, J=6 \mathrm{~Hz}, 2 \mathrm{H}), 5.18(\mathrm{~m}, 2 \mathrm{H}), 5.89(\mathrm{~m}, 1 \mathrm{H})$, $7.13(\mathrm{~d}, J=8.4 \mathrm{~Hz}, 2 \mathrm{H}), 7.32(\mathrm{~d}, J=8.4 \mathrm{~Hz}, 2 \mathrm{H}), 7.95(\mathrm{~d}, J=3 \mathrm{~Hz}, 1 \mathrm{H}), 8.58(\mathrm{~s}, 1 \mathrm{H}) .13 \mathrm{C}-\mathrm{NMR}\left(\mathrm{CDCl}_{3}\right.$, $125 \mathrm{MHz}) \delta 12.1,30.1,108.2,118.0,121.2,124.2,128.4,129.0,131.1,132.8,132.9,134.5,156.0,166.0$. HRMS (ESI-TOF) $m / z$ : Calcd. for $\mathrm{C}_{17} \mathrm{H}_{15} \mathrm{ClN}_{3} \mathrm{O}_{3}{ }^{+}\left([\mathrm{M}+\mathrm{H}]^{+}\right)$344.0796. Found: 344.0797.

5-(4-(4-Chlorophenyl)-5-phenyl-1H-pyrrol-3-yl)-3-methyl-4-nitroisoxazole (3db). Green solid, yield 81\%, m.p. $257-259{ }^{\circ} \mathrm{C} .{ }^{1} \mathrm{H}-\mathrm{NMR}$ (DMSO- $\left.d_{6}, 600 \mathrm{MHz}\right) \delta 2.44(\mathrm{~s}, 3 \mathrm{H}), 7.14(\mathrm{~d}, J=8.5 \mathrm{~Hz}, 2 \mathrm{H}), 7.22-7.25$ $(\mathrm{m}, 3 \mathrm{H}), 7.30-7.33(\mathrm{~m}, 4 \mathrm{H}), 7.98(\mathrm{~d}, J=2 \mathrm{~Hz}, 1 \mathrm{H}), 12.40(\mathrm{~s}, 1 \mathrm{H}) .{ }^{13} \mathrm{C}-\mathrm{NMR}$ (DMSO- $\left.d_{6}, 150 \mathrm{MHz}\right) \delta 12.2$, $108.5,120.5,126.3,127.7,127.8,128.1,128.8,129.1,131.2,131.7,132.0,132.3,134.2,156.3,166.2$. HRMS (ESI-TOF) $\mathrm{m} / z$ : Calcd. for $\mathrm{C}_{20} \mathrm{H}_{15} \mathrm{ClN}_{3} \mathrm{O}_{3}{ }^{+}\left([\mathrm{M}+\mathrm{H}]^{+}\right)$380.0796. Found: 380.0792 .

5-(5-Benzyl-4-(4-chlorophenyl)-1H-pyrrol-3-yl)-3-methyl-4-nitroisoxazole (3eb). Green solid, yield 78\%, m.p. 197-199 ${ }^{\circ} \mathrm{C} .{ }^{1} \mathrm{H}-\mathrm{NMR}\left(\mathrm{CDCl}_{3}, 600 \mathrm{MHz}\right) \delta 2.52(\mathrm{~s}, 3 \mathrm{H}), 3.93(\mathrm{~s}, 2 \mathrm{H}), 7.14(\mathrm{~d}, J=7.2 \mathrm{~Hz}, 2 \mathrm{H}), 7.18-7.19$ $(\mathrm{m}, 2 \mathrm{H}), 7.26(\mathrm{~d}, J=14.4 \mathrm{~Hz}, 1 \mathrm{H}), 7.31-7.34(\mathrm{~m}, 4 \mathrm{H}), 7.91(\mathrm{~d}, J=3 \mathrm{~Hz}, 1 \mathrm{H}), 8.43(\mathrm{~s}, 1 \mathrm{H}) .{ }^{13} \mathrm{C}-\mathrm{NMR}$ $\left(\mathrm{CDCl}_{3}, 125 \mathrm{MHz}\right) \delta 12.6,31.8,108.2,121.6,124.5,127.0,128.5,128.6,129.0,130.2,131.2,132.9,133.0$, 137.9, 156.0, 166.0. HRMS (ESI-TOF) $m / z$ : Calcd. for $\mathrm{C}_{21} \mathrm{H}_{17} \mathrm{ClN}_{3} \mathrm{O}_{3}{ }^{+}\left([\mathrm{M}+\mathrm{H}]^{+}\right)$394.0953. Found: 394.0950.

5-(4-(4-Chlorophenyl)-5-(4-methylbenzyl)-1H-pyrrol-3-yl)-3-methyl-4-nitroisoxazole (3fb). Green solid, yield 83\%, m.p. $167-169{ }^{\circ} \mathrm{C} .{ }^{1} \mathrm{H}-\mathrm{NMR}\left(\mathrm{CDCl}_{3}, 600 \mathrm{MHz}\right) \delta 2.33(\mathrm{~s}, 3 \mathrm{H}), 2.52(\mathrm{~s}, 3 \mathrm{H}), 3.88(\mathrm{~s}, 2 \mathrm{H}), 7.03$ $(\mathrm{d}, J=7.8 \mathrm{~Hz}, 2 \mathrm{H}), 7.13(\mathrm{~d}, J=7.8 \mathrm{~Hz}, 2 \mathrm{H}), 7.19(\mathrm{~d}, J=8.4 \mathrm{~Hz}, 2 \mathrm{H}), 7.33(\mathrm{~d}, J=7.8 \mathrm{~Hz}, 2 \mathrm{H}), 7.90$ $(\mathrm{d}, J=3 \mathrm{~Hz}, 1 \mathrm{H}), 8.46(\mathrm{~s}, 1 \mathrm{H}) .{ }^{13} \mathrm{C}-\mathrm{NMR}\left(\mathrm{CDCl}_{3}, 125 \mathrm{MHz}\right) \delta 12.1,20.9,31.3,108.1,121.4,124.4$, 127.1, 128.4, 129.7, 130.6, 131.2, 132.9, 134.7, 136.7, 155.9, 166.00. HRMS (ESI-TOF) $m / z$ : Calcd. for $\mathrm{C}_{22} \mathrm{H}_{19} \mathrm{ClN}_{3} \mathrm{O}_{3}{ }^{+}\left([\mathrm{M}+\mathrm{H}]^{+}\right)$408.1109. Found: 408.1103 .

\subsection{Crystal Structure Determination}

Single crystal of 3ac, suitable for X-ray diffraction analysis, was obtained by slow evaporation of its solution in petroleum ether-EtOAc $(8: 1, v / v)$ at room temperature. Selected light green single crystal of 3ac was mounted on glass fibers. The intensity data were measured at $293 \mathrm{~K}$ on a Bruker SMART APEXII CCD; cell refinement: SAINT (Bruker, Billerica, MA, USA 2007); data reduction: SAINT; program(s) used to solve structure: SHELXS97 [51]; program(s) used to refine structure: SHELXL97 [51]; molecular graphics: SHELXTL [51]; software used to prepare material for publication: SHELXTL [51]. Crystallographic data for the structures 3ac have been deposited in the Cambridge Crystallography Data Centre (CCDC No. 1552332). 


\section{Conclusions}

In summary, we have developed an efficient tandem one-pot synthesis of the isoxazole-substituted pyrrole derivatives via [3+2] cycloaddition of TosMIC and analogs with various styrylisoxazoles. This reaction features high efficiency, mild reaction conditions, broad substrate scope, and readily available substrates. Further investigations on the bicyclization strategy of activated isocyanides for the divergent synthesis of complex architecture are currently underway in our laboratory.

Supplementary Materials: Supplementary data associated with this article can be found in the SI.

Acknowledgments: Financial support of this research provided by Science and Technology Planning Project of Jilin Province (20140204022NY, 20160414015GH) is greatly acknowledged.

Author Contributions: Xianxiu Xu and Dawei Zhang conceived and designed the experiments. Xueming Zhang performed the experiments. Dawei Zhang wrote the manuscript. Xianxiu Xu and Dawei Zhang revised the manuscript. All authors read and approved the final manuscript.

Conflicts of Interest: The authors declare no conflict of interest.

\section{References}

1. Bhardwaj, V.; Gumber, D.; Abbot, V.; Dhiman, S.; Sharma, P. Pyrrole: A resourceful small molecule in key medicinal hetero-aromatics. RSC Adv. 2015, 46, 15233-15266. [CrossRef]

2. Rane, R.A.; Sahu, N.U.; Gutte, S.D.; Mahajan, A.A.; Shah, C.P.; Bangalore, P. Synthesis and evaluation of novel marine bromopyrrole alkaloidbased hybrids as anticancer agents. Eur. J. Med. Chem. 2013, 63, 793-799. [CrossRef] [PubMed]

3. Gomha, S.M.; Eldebss, T.M.A.; Abdulla, M.M.; Mayhoub, A.S. Diphenylpyrroles: Novel p53 activators. Eur. J. Med. Chem. 2014, 82, 472-479. [CrossRef] [PubMed]

4. Sundberg, R.J.; Pearce, B.C. 3-(3-Pyrrolyl)thiopyrrolidones as precursors of benzo[1,2-b:4,3-b']dipyrroles. Synthesis of structures related to the phosphodiesterase inhibitors PDE-I and PDE-II. J. Org. Chem. 1985, 50, 425-432. [CrossRef]

5. Frederich, J.H.; Matsui, J.K.; Chang, R.O.; Harran, P.G. Substituted 2,2'-bipyrroles and pyrrolylfurans via intermediate isoxazolylpyrroles. Tetrahedron Lett. 2013, 54, 2645-2647. [CrossRef] [PubMed]

6. Jin, R.F.; Sun, W.D.; Tang, S.S. A DFT study of pyrrole-isoxazole derivatives as chemosensors for fluoride anion. Int. J. Mol. Sci. 2012, 13, 10986-10999. [CrossRef] [PubMed]

7. Yang, Z.P.; Zhang, K.; Gong, F.B.; Li, S.Y.; Chen, J.; Ma, J.S.; Sobenina, L.N.; Mikhaleva, A.I.; Yang, G.Q.; Trofimov, B.A. A new fluorescent chemosensor for fluoride anion based on a pyrrole-isoxazole derivative. Beilstein J. Org. Chem. 2011, 7, 46-52. [CrossRef] [PubMed]

8. Padmavathi, V.; Reddy, K.V.; Sarma, M.R.; Sumathi, R.P.; Reddy, D.B. 1,3-dipolar cycloaddition of dipolar reagents to bischalcones in the presence of Chloramine-T. Indian J. Chem. 2004, 43, 2238-2242. [CrossRef]

9. Sasada, T.; Sawada, T.; Ikeda, R.; Sakai, N.; Konakahara, T. Approach to trisubstituted 3-aminopyrrole derivatives by $\mathrm{Yb}(\mathrm{OTF})_{3}$-catalyzed [4+1] annulation of 2-azadiene with $\mathrm{Me}_{3} \mathrm{SiCN}$. Eur. J. Med. Chem. 2010, 22, 4237-4244. [CrossRef]

10. Chen, J.X.; Wu, H.Y.; Zheng, Z.G.; Jin, C.; Zhang, X.X.; Su, W.K. An approach to the Paal-Knorr pyrroles synthesis catalyzed by $\mathrm{Sc}(\mathrm{OTf})_{3}$ under solvent-free conditions. Tetrahedron Lett. 2006, 47, 5383-5387. [CrossRef]

11. Van Leusen, A.M.; Siderius, H.; Hoogenboom, B.E.; Van Leusen, D. A new and simple synthesis of the pyrrole ring system from Michael acceptors and Tosylmethylisocyanides. Tetrahedron Lett. 1972, 13, 5337-5340. [CrossRef]

12. Váradi, A.; Palmer, T.C.; Dardashti, R.N.; Majumdar, S. Isocyanide-based multicomponent reactions for the synthesis of heterocycles. Molecules 2016, 21, 19. [CrossRef] [PubMed]

13. Kaur, T.; Wadhwa, P; Sharma, A. Arylsulfonylmethyl isocyanides: A novel paradigmin organic synthesis. RSC Adv. 2015, 5, 52769-52787. [CrossRef]

14. Eckert, H. Diversity oriented syntheses of conventional heterocycles by smart multi component reactions (MCRS) of the last decade. Molecules 2012, 17, 1074-1102. [CrossRef] [PubMed]

15. Sadjadi, S.; Heravi, M.M. Recent application of isocyanides in synthesis of heterocycles. Tetrahedron 2011, 67, 2707-2752. [CrossRef] 
16. Lygin, A.V.; Meijere, A.D. Isocyanides in the synthesis of nitrogen heterocycles. Angew. Chem. Int. Ed. 2010, 49, 9094-9124. [CrossRef] [PubMed]

17. Tandon, V.K.; Rai, S. p-Toluenesulfonylmethyl isocyanide: A versatile synthon in organic chemistry. Sulfur Rep. 2003, 24, 307-385. [CrossRef]

18. Ugi, I.; Werner, B.; Dömling, A. The chemistry of isocyanides, their multicomponent reactions and their libraries. Molecules 2003, 8, 53-66. [CrossRef]

19. Lu, X.M.; Li, J.; Cai, Z.J.; Wang, R.; Wang, S.Y.; Ji, S.J. One pot synthesis of pyrrolo[3,4-c]quinolinone/ pyrrolo[3,4-c]quinolone derivatives from 2-aminoarylacrylates/2-aminochalcones and tosylmethyl isocyanide (TosMIC). Org. Biomol. Chem. 2014, 12, 9471-9477. [CrossRef] [PubMed]

20. Chen, W.T.; Shao, J.A.; Li, Z.; Giulianotti, M.A.; Yu, Y.P. Synthesis of 2,3,4-trisubstituted pyrroles via a facile reaction of vinyl azides and tosylmethyl isocyanide. Can. J. Chem. 2012, 90, 214-221. [CrossRef]

21. Poulard, C.; Cornet, J.; Legoupy, S.; Dujardin, G.; Dhal, R.; Huet, F. Synthesis of polysubstituted pyrroles. Lett. Org. Chem. 2009, 6, 359-361. [CrossRef]

22. Chang, J.H.; Shin, H. Practical one-pot syntheses of ethyl 4-substituted-1H-pyrrole-3-carboxylates from aldehydes. Org. Process Res. Dev. 2008, 12, 291-293. [CrossRef]

23. Krishna, P.R.; Ramana Reddy, V.V.; Srinivas, R. A new synthetic route to oxazole and pyrrole 2-deoxy-C-ribosides. Tetrahedron 2007, 63, 9871-9880. [CrossRef]

24. Santo, R.D.; Costi, R.; Massa, S.; Artico, M. Pyrrole-annulated heterocyclic systems. Synthesis of $2 H$-pyrrolo[3,4-b][1,5]benzothiazepine 4,4-dioxide derivatives. Synth. Commun. 1998, 28, 2517-2530. [CrossRef]

25. Pavri, N.P.; Trudell, M.L. An efficient method for the synthesis of 3-arylpyrroles. J. Org. Chem. 1997, 62, 2649-2651. [CrossRef] [PubMed]

26. Dhanalakshmi, P.; Shanmugam, S. Convenient one-pot multicomponent strategy for the synthesis of 6-pyrrolylpyrimidines. RSC Adv. 2014, 4, 29493-29501. [CrossRef]

27. Padmavathi, V.; Premakumari, C.; Venkatesh, B.C.; Padmaja, A. Synthesis and antimicrobial activity of amido linked pyrrolyl and pyrazolyl-oxazoles, thiazoles and imidazoles. Eur. J. Med. Chem. 2011, 46, 5317-5326. [CrossRef] [PubMed]

28. Donohoe, T.J.; Guyo, P.M.; Harji, R.R.; Heiliwell, M. The birch reduction of 3-substituted pyrroles. Tetrahedron Lett. 1998, 39, 3075-3078. [CrossRef]

29. Kumar, K.; More, S.S.; Goyal, S.; Gangar, M.; Khatik, G.L.; Rawal, R.K.; Nair, V.A. A convenient synthesis of 4-alkyl-3-benzoylpyrroles from $\alpha, \beta$-unsaturated ketones and tosylmethyl isocyanide. Tetrahedron Lett. 2016, 57, 2315-2319. [CrossRef]

30. Divakar, M.A.; Shanmugam, S. Live cell imaging of bacterial cells: Pyrenoylpyrrole-based fluorescence labeling. Chem. Biol. Drug Des. 2017. [CrossRef] [PubMed]

31. Hormaza, A.; Perez, O.F.A. Síntesis de una nueva serie de pirroles vía cicloadición. Rev. Soc. Quím. Perú 2009, 75, 12-16.

32. Surya PrakashRao, H.; Sivakumar, S. Aroylketene dithioacetal chemistry: Facile synthesis of 4-aroyl-3-methylsulfanyl-2-tosylpyrroles from aroylketene dithioacetals and TosMIC. Beilstein J. Org. Chem. 2007, 3, 1-5.

33. Dannhardt, G.; Kiefer, W.; Krämer, G.; Maehrlein, S.; Nowe, U.; Fiebich, B. The pyrrole moiety as a template for COX-1/COX-2 inhibitors. Eur. J. Med. Chem. 2000, 35, 499-510. [CrossRef]

34. Qiu, F.L.; Wu, J.W.; Zhang, Y.H.; Hu, M.; Yu, F.; Zhang, G.L.; Yu, Y.P. A novel synthesis of multisubstituted pyrroles via trisubstituted olefins and TosMIC derivatives. Lett. Org. Chem. 2012, 9, 305-308.

35. Dijkstra, H.P.; Have, R.T.; Van Leusen, A.M. A direct synthesis of 2-(trimethylstannyl)pyrroles from Michael acceptors and stannylated tosylmethyl Isocyanide. J. Org. Chem. 1998, 63, 5332-5338. [CrossRef]

36. Smith, N.D.; Huang, D.; Cosford, N.D.P. One-step synthesis of 3-aryl- and 3,4-diaryl-(1H)-pyrroles using tosylmethyl Isocyanide (TosMIC). Org. Lett. 2002, 20, 3537-3539. [CrossRef]

37. Chauhan, P.; Kaya, U.; Enders, D. Advances in organocatalytic 1,6-addition reactions: Enantioselective construction of remote stereogenic centers. Adv. Synth. Catal. 2017, 359, 888-912. [CrossRef]

38. Liu, K.; Xiong, Y.; Wang, Z.F.; Tao, H.Y.; Wang, C.J. Ligand-controlled stereodivergent 1,3-dipolar cycloaddition of azomethine ylides with 3-methyl-4-nitro-5-styrylisoxazoles. Chem. Commun. 2016, 52, 9458-9461. [CrossRef] [PubMed] 
39. Liu, X.W.; Yao, Z.; Yang, J.; Chen, Z.Y.; Liu, X.L.; Zhao, Z.; Lu, Y.; Zhou, Y.; Cao, Y. 1,3-Dipolar cycloaddition enabled isoxazole-fused spiropyrrolidine oxindoles syntheses from 3-methyl-4-nitro-5-alkenyl-isoxazoles and azomethine ylides. Tetrahedron 2016, 72, 1364-1374. [CrossRef]

40. Liu, X.L.; Han, W.Y.; Zhang, X.M.; Yuan, W.C. Highly efficient and stereocontrolled construction of 3,3'-pyrrolidonyl spirooxindoles via organocatalytic domino michael/cyclization reaction. Org. Lett. 2013, 15, 1246-1249. [CrossRef] [PubMed]

41. Disetti, P.; Moccia, M.; Illera, D.S.; Suresh, S.; Adamo, M.F.A. Catalytic enantioselective addition of isocyanoacetate to 3-methyl-4-nitro-5-styrylisoxazoles under phase transfer catalysis conditions. Org. Biomol. Chem. 2015, 13, 10609-10612. [CrossRef] [PubMed]

42. Zhang, X.; Feng, C.J.; Jiang, T.; Li, Y.F.; Pan, L.; Xu, X.X. Expedient and divergent tandem one-pot synthesis of benz[e]indole and spiro[indene-1,3'-pyrrole] derivatives from alkyne-tethered chalcones/cinnamates and TosMIC. Org. Lett. 2015, 17, 3576-3579. [CrossRef] [PubMed]

43. Du, J.; Xu, X.X.; Li, Y.F.; Pan, L.; Liu, Q. [3+3]-cycloaddition reactions of $\alpha$-acidic isocyanides with 1,3-dipolar azomethine lmines. Org. Lett. 2014, 16, 4004-4007. [CrossRef] [PubMed]

44. Zhang, L.J.; Zhang, X.M.; Lu, Z.C.; Zhang, D.W.; Xu, X.X. Accessing benzo[f]indole-4,9-diones via a ring expansion strategy: Silver-catalyzed tandem reaction of tosylmethyl isocyanide (TosMIC) with 2-methyleneindene-1,3-diones. Tetrahedron 2016, 72, 7926-7930. [CrossRef]

45. Li, Y.F.; Xu, X.X.; Shi, H.; Pan, L.; Liu, Q. Bicyclization of isocyanides with alkenoyl bis(ketene dithioacetals): Access to 6,7-dihydro-1H-indol-4(5H)-ones. J. Org. Chem. 2014, 79, 5929-5933. [CrossRef] [PubMed]

46. Zhang, L.J.; Xu, X.X.; Tan, J.; Pan, L.; Xia, W.M.; Liu, Q. Tandem Michael addition/intramolecular isocyanide [3+2] cycloaddition: Highly diastereoselective one pot synthesis of fused oxazolines. Chem. Commun. 2010, 46, 3357-3359. [CrossRef] [PubMed]

47. Zhang, L.J.; Xu, X.X.; Xia, W.M.; Liu, Q. Bicyclization of isocyanides: A synthetic strategy for fused pyrroles. Adv. Synth. Catal. 2011, 353, 2619-2623. [CrossRef]

48. Qiu, F.L; Wu, J.W.; Zhang, Y.H.; Hu, M.; Yu, Y.P. One-pot cascade approach to 1,3'-bipyrrole derivatives from trisubstituted olefins with tosylmethyl-isocyanide (TosMIC). Tetrahedron Lett. 2012, 53, 446-448. [CrossRef]

49. Zhang, J.L.; Liu, X.H.; Ma, X.J.; Wang, R. Organocatalyzed asymmetric vinylogous Michael addition of $\alpha, \beta$-unsaturated $\gamma$-butyrolactam. Chem. Commun. 2013, 49, 9329-9331. [CrossRef] [PubMed]

50. Magnus, P.; Gallagher, T.; Schultz, J.; Or, Y.S.; Ananthanarayan, T.P. Studies on the synthesis of the antitumor agent CC-1065: Synthesis of the unprotected cyclopropapyrroloindole A portion using the 3,3'-bipyrrole strategy. J. Am. Chem. Soc. 1987, 109, 2706-2711. [CrossRef]

51. Sheldrick, G.M. A short history of SHELX. Acta Cryst. 2008, 64, 112-122. [CrossRef] [PubMed]

Sample Availability: Samples of the compounds $3 \mathbf{a a}-\mathbf{f b}$ are available from the authors.

(C) 2017 by the authors. Licensee MDPI, Basel, Switzerland. This article is an open access article distributed under the terms and conditions of the Creative Commons Attribution (CC BY) license (http:/ / creativecommons.org/licenses/by/4.0/). 